\title{
Electromyography (EMG)-signal based fuzzy-neuro control of a 3 degrees of freedom (3DOF) exoskeleton robot for human upper-limb motion assist ${ }^{\star \star}$
}

\author{
R.A.R.C. Gopura* and Kazuo Kiguchi \\ Department of Advanced Systems Control Engineering, Graduate School of Science and Engineering, Saga University, Saga, Japan.
}

\begin{abstract}
An electromyography (EMG) signal based fuzzyneuro control method is proposed in this paper for a human upper-limb motion assist exoskeleton robot. The upper-limb exoskeleton robot (named W-EXOS) assists the motions of human forearm pronation/supination, wrist flexion/extension and ulnar/radial deviation. The paper presents the EMG signal based fuzzy-neuro control method with multiple fuzzy-neuro controllers and the adaptation method of the controllers. The skin surface EMG signals of muscles in the forearm of the exoskeleton user and the hand force/forearm torque measured from the sensors of the exoskeleton robot are used as input information for the controllers. Fuzzy-neuro control method, which is a combination of flexible fuzzy control and adaptive neural network control, has been applied to realize the natural and flexible motion assist. In the control method, multiple fuzzy-neuro controllers are applied, since the muscle activation levels change in accordance with the angles of motions. The control method is able to adapt in accordance with the changing EMG signal levels of different users. Experiments have been performed to evaluate the proposed control method.
\end{abstract}

Keywords: Exoskeleton robot, fuzzy-neuro control, human performance augmentation, power-assist.

\section{INTRODUCTION}

An exoskeleton robot is an orthotic device which has an external structural mechanism with links and joints. When it is worn by a human user its joints and links correspond to the user's joints and limbs. The exoskeleton robot transmits torques to the joints of the user from the actuators through its links. The exoskeleton robot can be used in applications such as a rehabilitation device, an assistive device, a human-amplifier or a haptic interface.
Currently, the birthrate is decreasing and the people enjoy longer life because of the increase in life expectancy ${ }^{1}$. Therefore, the working population is decreasing and also taking care of physically weak individuals (aged, injured and/or differently abled person) is becoming a problem. Sri Lanka has a considerable number of injured and/or differently abled individuals. The number increased due to the war. In this situation, robotic technology plays an important role in the field of welfare and rehabilitation. Several exoskeleton robots and their control methods have been studied in previous research ${ }^{2-18}$ to assist the daily upper-limb motions and/or rehabilitation of physically weak individuals such as elderly, injured, or differently abled. The shoulder, elbow, and wrist motions are important for people to perform daily activities. This paper presents an EMG signal based fuzzy-neuro control method for an upper-limb exoskeleton robot (W-EXOS) ${ }^{9-10}$ for human wrist flexion/extension, ulnar/ radial deviation and forearm pronation/supination motion assist. The EMG signals are important biological signals which are generated when muscles are activated.

The control methods of exoskeleton robots are different according to the purpose of the robot. There are many control methods (i.e. ARMin ${ }^{5}$-impedance control, L-EXOS ${ }^{13}$-force control) for the exoskeleton robots. In the $\mathrm{ARMin}^{5}$, an adjustable impedance controller is used for assisting the subject in catching a ball where the assisting force is proportional to the horizontal distance between the hand and the ball and the impedance parameters are set depending on the vertical position of the ball. In the L-EXOS ${ }^{13}$, the desired value of force is computed through

\footnotetext{
* Corresponding author (gopura@ieee.org)

** Part of this work was presented at the IEEE International Conference on Robotics and Automation, 2008.
} 


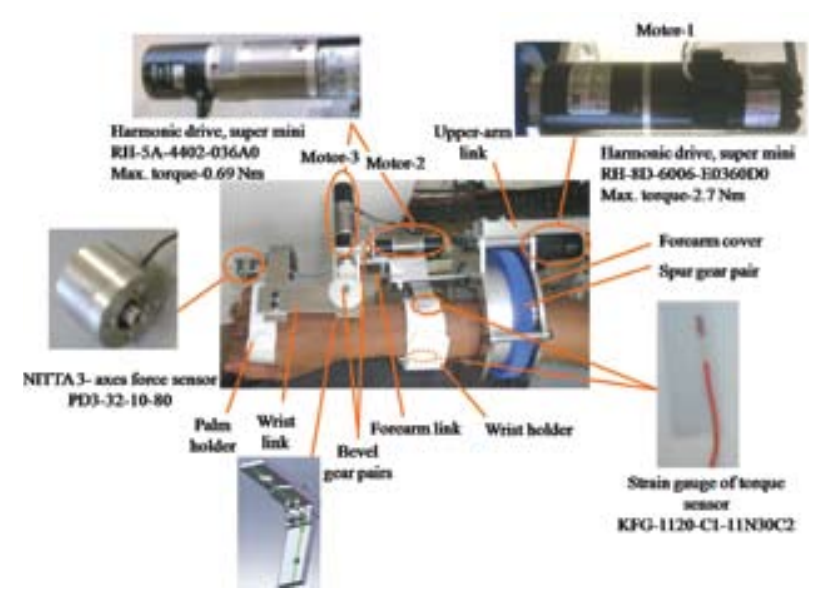

Figure 1: W-EXOS: 3DOF wrist and forearm motion assist exoskeleton robot

the estimation of a desired position for the end effecter and the desired position through a constraint-based algorithm. Since the EMG signals of human muscles directly reflect the human motion intension, they can be used as input information for the control of robotic systems ${ }^{9-12,19-21}$. The skin surface EMG signals of muscles in forearm of the user of exoskeleton robot are used as main input information for the proposed controller. The hand force (i.e. generated force between the robot and the hand of the robot user when the motions of wrist flexion/extension and/or ulnar/radial deviation are performed) and forearm torque (i.e. generated torque between the wrist holder of the robot and the forearm of robot user when the forearm supination/pronation motion is performed) are also used as subordinate input information for the controller. By applying the skin surface EMG signals as main input signals to the controller, automatic power-assist can be realized for the physically weak persons without manipulating any other equipment.Such kind of control is especially important for the system used by physically weak individuals. Since the forearm consists of many kinds of muscles which are involved in many motions ${ }^{22,}$ ${ }^{23}$, it is not easy to apply EMG signals of muscles of the forearm as input signals to the controller. In addition, EMG signal based control is difficult to be realized for multi-degrees of freedom (DOF) in power-assist exoskeleton robots ${ }^{11}$. Moreover, the effect of the change of working muscle activation levels in accordance with the angles of motions should be considered. Therefore, a fuzzy-neuro control method with multiple controllers ${ }^{12}$ is proposed for the control of the exoskeleton robot to deal with the above mentioned difficulties and to generate natural and flexible forearm and wrist motions. In this study, fuzzy IF-THEN control rules are defined for forearm and wrist motions. By applying defined fuzzy IF-THEN control rules, nine fuzzy-neuro controllers are designed for forearm and wrist flexion/extension motion and another fuzzy-neuro controller is designed for wrist radial/ulnar deviation. In the proposed control method, multiple fuzzy-neuro controllers are switched moderately according to the angles of forearm and wrist motions ${ }^{12}$. Multiple controllers are necessary since activation levels of the muscles change according to the angles of forearm and wrist motions. In this paper, controller adaptation to EMG signals of the user is also discussed. Because of the adaptation ability of the fuzzy-neuro controllers, the exoskeleton robot is flexible enough to deal with EMG signals. Experiments have been performed to evaluate the proposed control method.

\section{W-EXOS: 3DOF forearm and wrist motion assist exoskeleton robot}

The W-EXOS is shown in Figure 1. It has been developed to assist the motions of forearm pronation/supination, wrist flexion/extension and ulnar/radial deviation ${ }^{9,10}$. The original feature of the design is the axes offset of wrist joint, which was not considered in any other design. Therefore, undesired pain of the user's wrist joint can be avoided. In addition, the hand-robot interface has been designed so as not to disturb the finger motions. The axes offset of wrist joint is important for the wrist joint of the exoskeleton robot, since the wrist joint is sensitive to change in position and torque. The W-EXOS
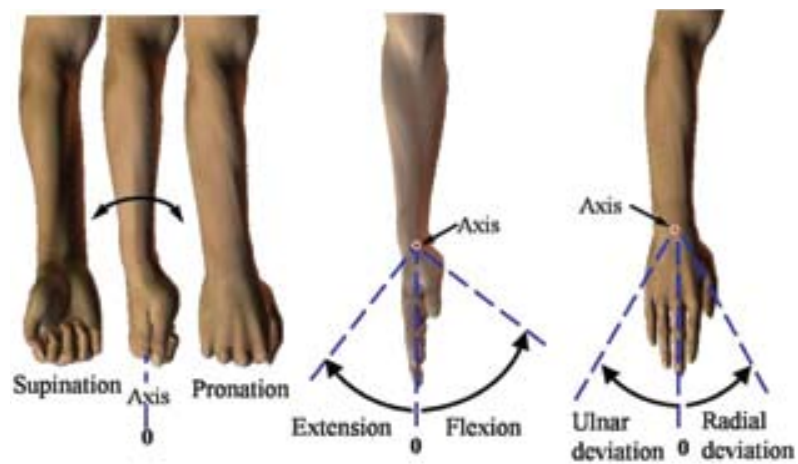

Figure 2: Assisting motions of the W-EXOS. (a) Forearm pronation/ supination motion, (b) wrist flexion/extension motion and (c) wrist radial/ulnar deviation

Table 1: Movable ranges of exoskeleton robot and human upper limb

\begin{tabular}{lcc}
\hline Motion & $\begin{array}{c}\text { Movable range of } \\
\text { exoskeleton [deg] }\end{array}$ & $\begin{array}{c}\text { Average movable range } \\
\text { of human [deg] }\end{array}$ \\
\hline Radial deviation & 20 & 25 \\
Ulnar deviation & 30 & 35 \\
Flexion & 60 & 70 \\
Extension & 50 & 60 \\
Pronation & 60 & 75 \\
Supination & 80 & 80 \\
\hline
\end{tabular}




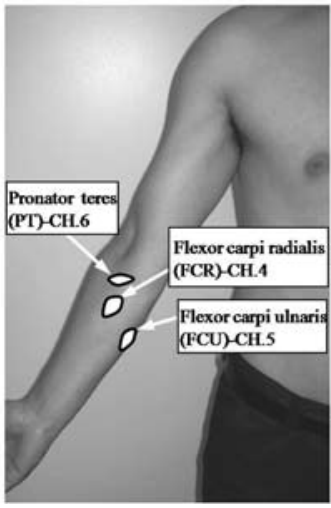

(a)

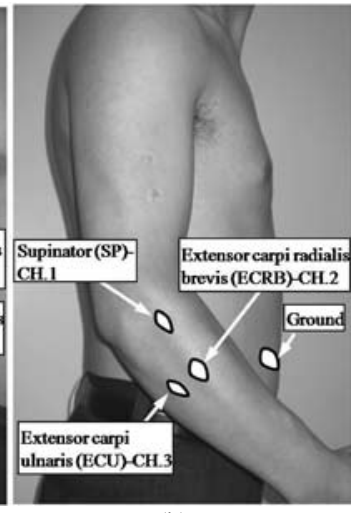

(b)
Figure 3: Locations of EMG electrodes. (a) Front view. (b) Side view.

mainly consists of forearm motion support part and wrist motion support part. The forearm motion support part consists of two links (upper-arm link and forearm link), a direct current (DC) motor, a drive and driven spur gear pair (gear ratio 1:3), a wrist holder, a forearm cover and torque sensors. The wrist motion support part consists of a link attachment, two DC motors, two drive and driven bevel gear pairs (gear ratio-1:2), a palm holder, a three axis force sensor and a link (wrist link) which connects the palm holder and link attachment as shown in Figure 1. Proximal end of the Link-1 of the W-EXOS can be attached to the upper-limb exoskeleton robot $^{2}$ which is installed on a mobile wheel chair that can be used by many physically weak persons. Therefore, the user does not feel the weight of the exoskeleton robot at all. The details about the design and the mechanism of the W-EXOS can be referred ${ }^{10}$. Assisting motions of the $\mathrm{W}$-EXOS is shown in Figure 2. Considering the safety of the user and the required motion in daily activities, the limitation of the movable ranges of the exoskeleton robot is set as shown in Table 1. Since the W-EXOS is directly in contact with the human user, safety is essential. Mechanical stoppers are attached for each motion to prevent the exceeding of the movable range. The robot does not include any sharp edges in its mechanical construction and mechanical stoppers. Furthermore, each motor has an individual switch and there is an emergency stop switch beside the exoskeleton robot.

\section{METHODS AND MATERIALS}

EMG signal based controller: The input signals for the proposed control method are EMG signals and/or the hand force/forearm torque. The EMG signals are biological signals which are generated when muscles are activated. It directly reflects the human motion intention. The amplitude of EMG signal is $0.01-10 \mathrm{mV}$ and the frequency is $10-2000 \mathrm{~Hz}$. In the control method, the motion assist is carried out based on the EMG activity levels and/ or the hand force/forearm torque according to the fuzzy rules of the controller. The forearm consists of many kinds of muscles, which are involved in finger motion, forearm pronation/supination motion, and elbow motion as well as wrist motion. Some muscles are used for two motions (i.e. flexor carpi ulnaris for wrist flexion motion and ulnar deviation and extensor carpi radialis brevis for wrist extension and radial deviation). Therefore, it is not easy to separate wrist motions of the user, based on the EMG signals of the related muscles. Among the muscles that activate the wrist and forearm motions, there is an overlap in some muscles. The surface anatomy of each muscle is studied and the location where only the desired muscle is exposed to skin is selected as the location for the electrode of the particular muscle. Six kinds of EMG signals are measured with electrodes (NE-101A, Nihon Koden Co.) through an amplifier (MEG-6108, Nihon Koden Co.) to control the motions of the W-EXOS. They are CH. 1: supinator (SP), CH. 2: extensor carpi radialis brevis (ECRB), CH. 3: extensor carpi ulnaris (ECU), $\mathrm{CH}$. 4: flexor carpi radialis (FCR), CH. 5: flexor carpi ulnaris (FCU) and $\mathrm{CH}$. 6: pronator teres (PT). Table 2 shows the muscles and the related EMG signal channels used to activate the motions of the W-EXOS. The locations of EMG electrodes are shown in Figure 3. Because of the difficulty in using raw data of EMG signals for input information of the controller, features have to be extracted from the raw EMG signal data. In this study, Root Mean Square (RMS) has been applied to extract the feature of the raw EMG signal for the fuzzy-neuro controller. The equation of RMS is given as follows.

$R M S=\sqrt{\frac{1}{N} \sum_{i=1}^{N} v_{i}^{2}}$

Table 2: Muscles used for motions of W-EXOS

\begin{tabular}{lll}
\hline Motion & Used muscles & Channel \\
\hline \multirow{2}{*}{ Wrist flexion } & Flexor carpi radialis & CH. 4 \\
& Flexor carpi ulnaris & CH. 5 \\
Wrist extension & Extensor carpi radialis brevis & CH. 2 \\
& Extensor carpi ulnaris & CH. 3 \\
Wrist radial deviation & Extensor carpi radialis brevis & CH. 2 \\
& Flexor carpi radialis & CH. 4 \\
Wrist ulnar deviation & Extensor carpi ulnaris & CH. 3 \\
Forearm pronation & Flexor carpi ulnaris & CH. 5 \\
Forearm supination & Supinator & CH. 6 \\
\hline
\end{tabular}



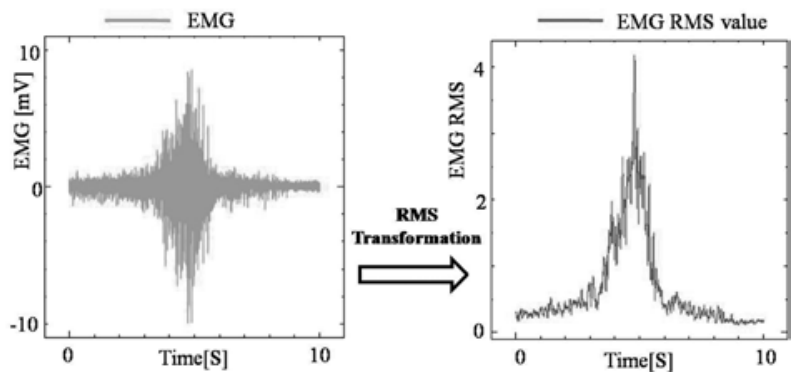

Figure 4: Example of EMG and RMS value of EMG

where, $v_{i}$ is the voltage value at the $i$ th sampling and $N$ is the number of sample in a segment. The number of samples is set to be 100 and the sampling frequency is $2 \mathrm{kHz}$ in this study. An example of EMG signals and their RMS is shown in Figure 4. In the control method, movable ranges of forearm pronation/supination and wrist flexion/extension motions are divided into three sections each and expressed by membership functions as shown in Figure 5. By expressing the range of each section with membership functions, the controller can be gradually switched according to the arm posture. The movable range of wrist radial/ulnar deviation is not divided into sections, since its movable range is comparatively narrow. A fuzzy-neuro (fuzzy rule structured neural network) controller is designed for each section. Therefore, nine fuzzy-neuro controllers are designed in total. For wrist radial/ulnar deviation motion, another fuzzy-neuro controller is applied considering the movable range of wrist radial/ulnar deviation as one section. The architecture of one of the nine fuzzy-neuro controller is shown in Figure 6(a) as an example. The architecture of the other controllers are also similar to that of the shown controller [Figure 6(a)] except the connection between the fuzzifier and rule layers. The fuzzy-neuro controller of wrist radial/ulnar deviation is shown in Figure 6(b). Each fuzzy-neuro controller consists of five layers (input layer, fuzzifier layer, rule layer, defuzzifier layer and output layer). In Figure $6, \Sigma$ is the sum of the inputs, $\Pi$ is the multiplication of the inputs, $F_{x}$ and $F_{z}$ are the force sensor signals for $x$ and $z$ axis and $\tau_{1}, \tau_{2}, \tau_{3}$ are controller output for the motors of forearm motion, wrist flexion/extension motion and wrist radial/ulnar deviation, respectively. Controller outputs are multiplied by the power-assist rate and the friction compensation value is added to obtain the torque commands for three motors. $f_{g}$ and $f_{s}$ are two kinds of nonlinear functions which are applied to express the membership function of the fuzzy-neuro controller.
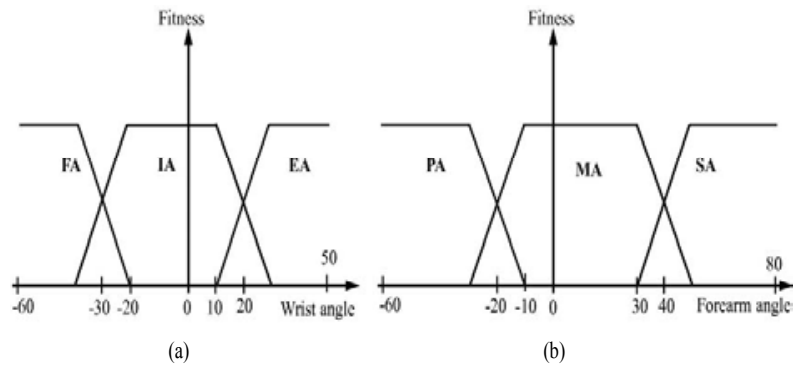

Figure 5: Membership functions of movable ranges. (a) Membership functions of movable range of wrist flexion/extension. Here, FA, IA, and EA are flexed angle, intermediate angle, and extended angle, respectively. (b) Membership functions of movable range of forearm supination/pronation. Here, PA, MA, and SA are pronated angle, middle angle, and supinated angle, respectively.

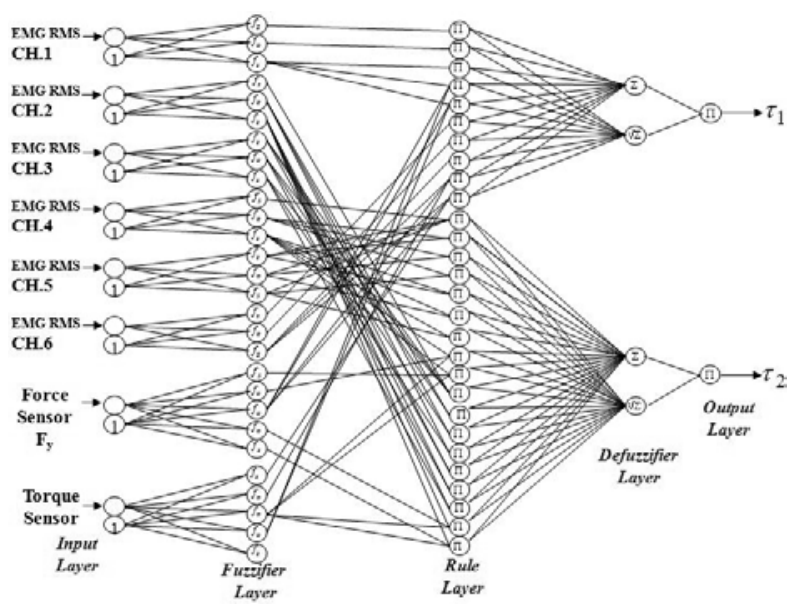

(a)

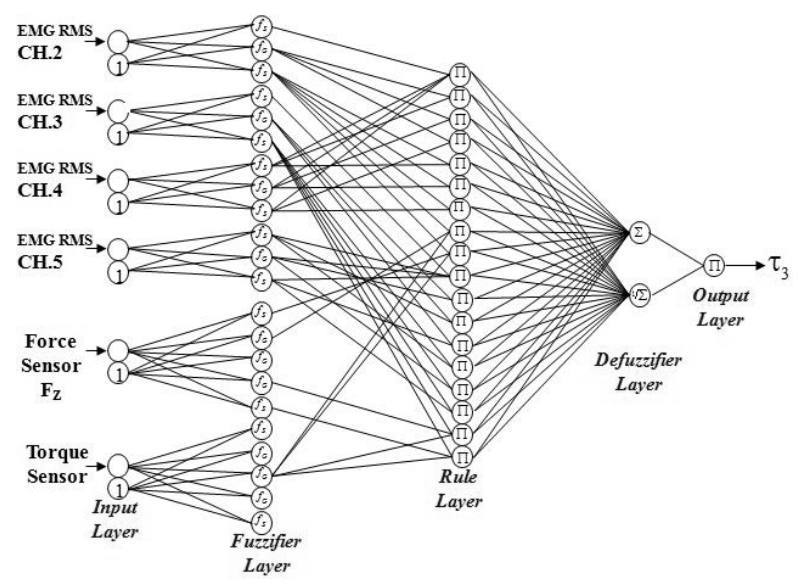

(b)

Figure 6: Architectures of the fuzzy-neuro controllers. (a) Architecture of one of the nine fuzzy-neuro controller. (b) Architecture of the wrist radial/ulnar deviation controller 


$$
\begin{aligned}
& f_{S}\left(u_{S}\right)=\frac{1}{1+e^{-u_{S}}} \\
& u_{S}(x)=w_{0}+w_{i} x \\
& f_{G}\left(u_{G}\right)=e^{-u_{G}^{2}} \\
& u_{G}(x)=\frac{w_{0}+x}{w_{i}}
\end{aligned}
$$

where $w_{o}$ is the threshold value and $w_{i}$ is the weight value. Fuzzy IF-THEN rules are defined for each fuzzyneuro controller ${ }^{10}$. Fuzzy IF-THEN control rules have been designed based on a preliminary experiment which was performed to find out the activity patterns of EMG signals of the muscles used for the forearm and wrist motions ${ }^{10}$. Fuzzy rules are designed to provide torque

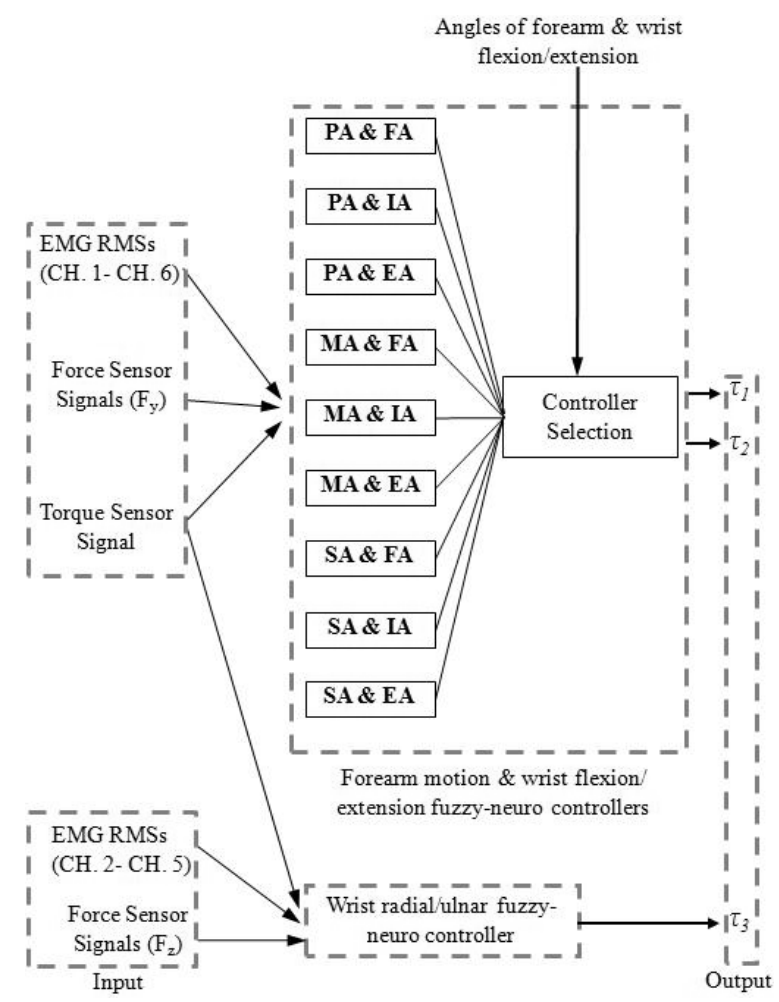

Figure 7: Structure of the proposed control method. Input information for the controllers is EMG RMSs, force/torque sensor signals, and angles of forearm and wrist flexion/extension. Outputs from the controllers are three kinds of torque commands. Forearm and wrist flexion/extension controller has nine fuzzy-neuro controllers. PA \& FA is the controller which is defined for the forearm pronated region and wrist flexed region depicted in Figure 5. Other controllers are also defined for the relevant regions indicated by their names commands according to the EMG RMSs of six muscles and force/torque sensor signals. In the fuzzy rules, the generated hand force/forearm torque are considered to be more reliable when the user activates the muscle a little (when the EMG levels of the user are low), and EMG signals are considered to be more reliable when the user activates the muscle a lot (when the EMG levels of the user are high). The structure of the proposed control method of the W-EXOS is shown in Figure 7. It consists of nine fuzzy-neuro controllers for the forearm motion and the wrist flexion/extension motion and another fuzzy-neuro controller for wrist radial/ulnar deviation. Input information for the nine fuzzy-neuro controllers are the EMG RMSs of six kinds of muscles (CH. 1-CH. 6), the force sensor signal and the torque sensor signal. In addition to the EMG RMSs and force/torque sensor signals, angles of forearm and wrist flexion/extension motions are given as additional input information for the nine controllers to switch the required controller according to the angles of the forearm and the wrist. Input information for the wrist radial/ulnar fuzzy-neuro controller is the EMG RMSs of four kinds of muscles (CH. 2-CH. 5), the force sensor signals and the torque sensor signal. Three kinds of variables (ZO: Zero, PS: Positive Small and PB: Positive Big) are prepared for each EMG RMSs. Five kinds of variables (NB: Negative Big, NS: Negative Small, ZO, PS and PB) are prepared for each sensor signals.

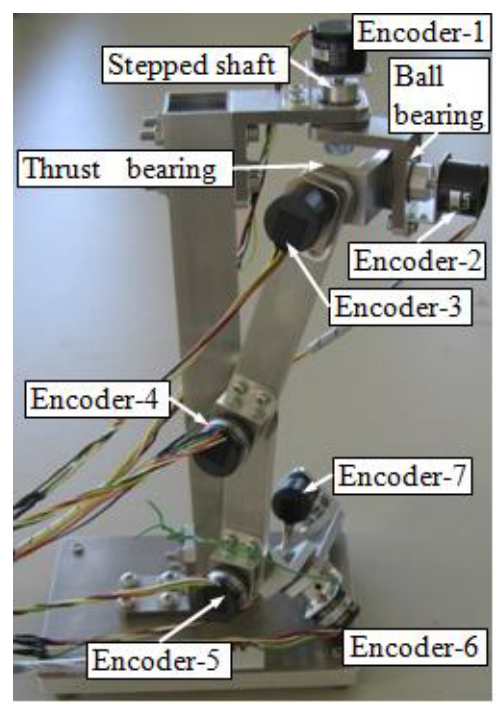

Figure 8: Teaching device. It has seven incremental encoders. In this study, encoder-5, encoder- 6 and encoder- 7 are used to indicate the motions for controller adaptation 


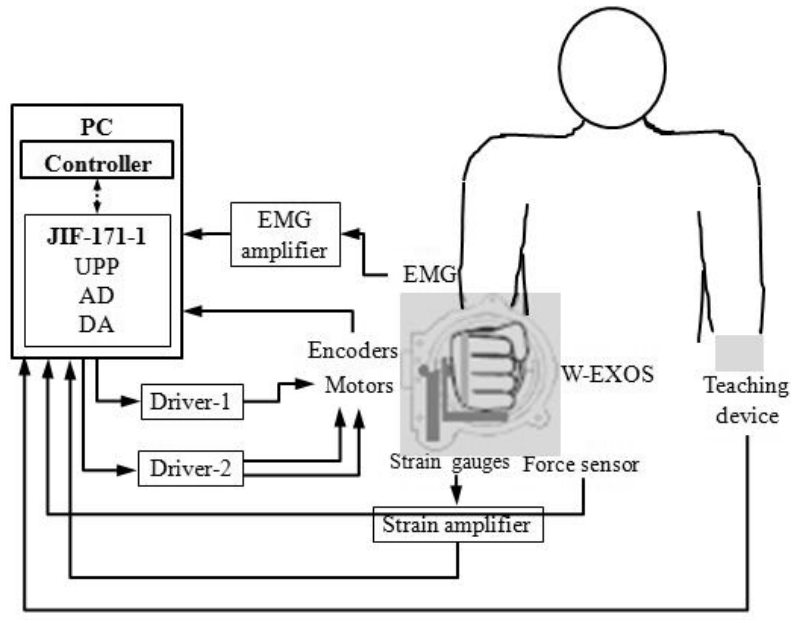

Figure 9: Experimental set-up that consists of the W-EXOS with the user, the teaching device, EMG amplifier, strain amplifier, two motor drives and personal computer with interface card (JIF-171-1)

Adaptation of the Controller: The adaptation of the controller to the conditions of the robot user is important, since the EMG signal is a biological signal ${ }^{11}$. The adaptation of fuzzy-neuro controllers are carried out by adjusting each weight value of the fuzzy-neuro controller to minimize the evaluation function. In this study, error back-propagation learning algorithm has been applied to minimize the squared error function. In order to indicate the user's motion intention correctly, a teaching device (Figure 8) that has the same DOF and the same link ratio as the exoskeleton robot is directly operated by the user with his/her un-assisted arm. The exoskeleton robot user is supposed to indicate the way of moving the assisted forearm and wrist joints (i.e. the correct user motion intention) using the teaching device. The teaching device consists of 7 encoders, ball bearings, thrust bearings, links, stepped shafts and a stand. Each encoder is connected to the device by using a stepped shaft, a ball bearing and a thrust bearing. The teaching device is designed to indicate the complete upper-limb motions. In this study, encoder- 5 , encoder- 6 and encoder- 7 are used to train of the wrist and forearm motions. The controller teaching is supposed to perform 1,2 minutes when the user's condition is changed. The evaluation function for the fuzzy-neuro controller training is given as follows.

$$
\mathrm{E}=1 / 2\left(\left(\theta_{d}-\theta\right)^{2}+\alpha \sum\left(R M S_{d}-R M S\right)^{2}\right)
$$

where $\theta_{d}$ is the desired joint angle indicated by the teaching device shown in Figure 8, $\theta$ is the joint angle of the exoskeleton, $\alpha$ is the rate of EMG adaptation and $R M S_{d}$ is the desired EMG level. The maximum value of each related muscle activity level is modelled as sinusoidal function in the beginning and ending of the motions and taken as $R M S_{d}$. This modelling eliminates the undesired errors of the $R M S_{d}$ in the beginning and in the ending of the motions. In the middle of the motions, the maximum value of each related activity level of muscle is taken as $R M S_{d}$.

\section{RESULTS}

Experiments were performed to evaluate the effectiveness of the proposed control method. Experimental set-up is shown in Figure 9. In the first experiment, a young male subject (29 years old) performed wrist and forearm motions with and without the assistance of the exoskeleton after the training of the controller. If the exoskeleton robot properly assists the motions, the muscle activation levels should be reduced with the motion assist. The activation level of muscle (raw EMG data) of flexor carpi ulnaris for wrist flexion motion is shown in Figure 10(a). From the activation level of muscle of flexor carpi ulnaris (CH. 5), it can be seen that the activation level was reduced when the robot assisted the motion. Another result of forearm pronation motion is shown in Figure 10(b). Here, the motion was performed from fully supinated position to fully pronated position. The EMG activity level of the muscle of pronator teres $(\mathrm{CH} .6)$ has been reduced when the exoskeleton robot assisted the motion. In the second experiment, another male subject ( 28 years old) performed wrist and forearm motions with the assistance
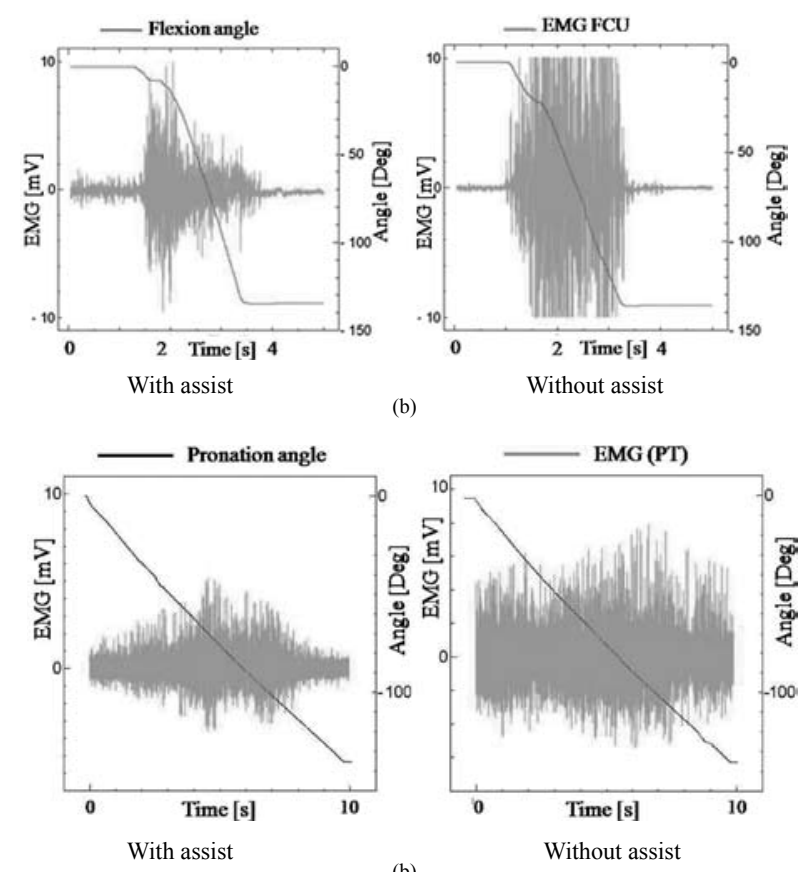

(b)

Figure 10: Experimental results for motion assist. (a) Wrist flexion motion (CH. 5) (b) Forearm pronation motion (CH.6) 


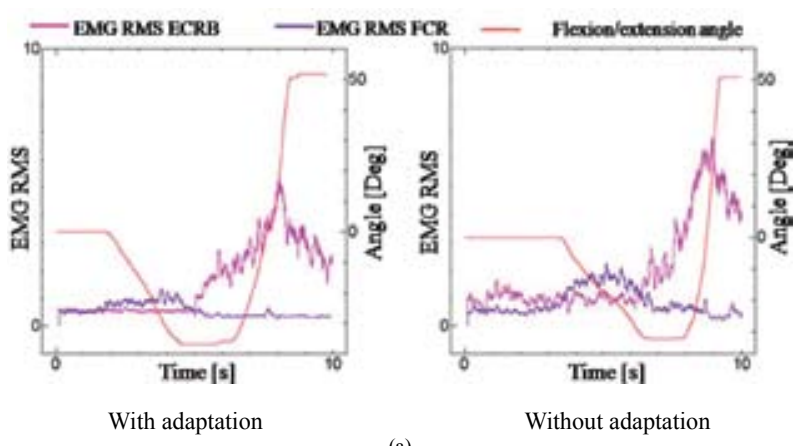

(a)

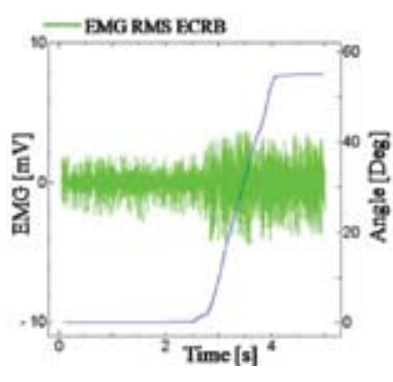

With adaptation

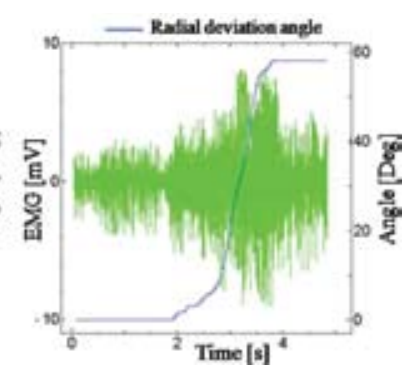

Without adaptation (b)

Figure 11: Experiment results for controller adaptation (a) Wrist flexion/extnsion motion (b) Wrist radial deviation

of the exoskeleton robot to show the adaptation ability of the controller. Figure 11(a) shows the experimental result for wrist flexion/extension motion with and without adaptation of the controller. It can be seen that the muscle activation level is reduced because of the adaptation of the controller. The experimental results for wrist radial deviation with and without adaptation of the controller are shown in Figure 11(b). From the results, one can see that the muscle activation is reduced after the adaptation of the controller to the user.

The experimental results (Figure 10) prove that the exoskeleton robot with the EMG-signal based fuzzyneuro controller can effectively assist human wrist and forearm motions. In addition, the adaptation ability of the controller to the user of the exoskeleton robot is proved by the experimental results (Figure 11).

\section{CONCLUSION}

The exoskeleton robot can be used in applications such as a rehabilitation device, an assistive device, a humanamplifier and a haptic interface. In a society with a higher percentage of aged population, the exoskeleton robots can be used specially for motion assist and rehabilitation of injured and/or differently abled persons.
In this paper, an EMG-signal based fuzzy-neuro control method is proposed for a 3DOF upper-limb exoskeleton robot. In the control method, multiple fuzzy-neuro controllers are switched moderately according to the angles of the forearm and the wrist. The proposed control method is able to adapt itself to the condition of the user. The experimental results show that the W-EXOS is able to assist wrist and forearm motion of the user with the EMG-based fuzzy-neuro control method. In addition, the smooth forearm pronation/supination motion, wrist motions and daily motions are generated by the W-EXOS with the proposed control method in accordance with the user's motion intention.

\section{Acknowledgement}

This work was supported by the Japan Society for the Promotion of Science (JSPS) Grant-in-Aid for Scientific Research (C) 19560258.

\section{References}

1. www.un.org/esa/population/publications/worldageing 19502050/. Accessed in February 2009.

2. Kiguchi K., Kariya S., Watanabe K., Izumi K. \& Fukuda T. (2001). An exoskeletal robot for human elbow motion support-sensor fusion, adaptation, and control. IEEE Transactions on Systems, Man, and Cybernetics, Part B 31(3): 353-361.

3. Kiguchi K. (2007). Active exoskeletons for upperlimb motion assist. Journal of Humanoid Robotics 4(3): 607-624.

4. Lemay M.A., Hogan N. \& van Dorsten J.W.A. (1998). Issue in impedance selection and input devices for multi-joint powered orthotics. IEEE Transactions on Rehabilitation Engineering 6(1): 102-105.

5. Nef T., Mihelj M., Colombo G. \& Riener R. (2006). ARMin Robot for rehabilitation of the upper extremities. Proccedings of the IEEE International Conference on Robotics and Automation, May 2006, Florida, USA: 31523157.

6. Sasaki D., Noritsugu T. \& Takaiwa M. (2004). Development of active support splint driven by pneumatic soft actuator (ASSIST). Journal of Robotics and Mechatronics 16: 497502.

7. Perry J.C. \& Rosen J. (2007). Upper-limb powered exoskeleton design. IEEE/ASME Transactions on Mechatronics 12(4): 408-417.

8. Tsagarakis N.G. \& Caldwell D.C. (2003). Development and control of a soft-actuated exoskeleton for use in physiotherapy and training. Journal of Autonomous Robots 15: 21-33.

9. Gopura R.A.R.C. \& Kiguchi K. (2008). EMG-based control of an exoskeleton robot for human forearm and wrist motion assist. Proccedings of the IEEE International Conference on Robotics and Automation, May, 2008, California, USA : 731-736. 
10. Gopura R.A.R.C. \& Kiguchi K. (2008). An exoskeleton robot for human forearm and wrist motion assist-hardware design and EMG-based controller. Journal of Advanced Mechanical Design, Systems, and Manufacturing 2(6): 1067-1083.

11. Kiguchi K., Imada Y. \& Liyanage M. (2007). EMG-based neuro-fuzzy control of a 4DOF upper-limb power-assist exoskeleton. Proceedings of the International Conference of the IEEE Engineering in Medicine and Biology Society August, 2007, Yon, France, 3040-3043.

12. Kiguchi K., Kariya S., Watanabe K. \& Fukuda T. (2002). Application of multiple fuzzy-neuro controllers of an exoskeletal robot for human elbow motion support. Transaction on Control, Automation and Systems Engineering 4(1): 49-55.

13. Frisoli A., Rocchi F., Marcheschi S., Dettori A., Salsedo F. \& Bergamasco M. (2005). A new force-feedback arm exoskeleton for haptic interaction in virtual environments. Proceedings of the First Eurohaptics Conference and Symposium on Haptic Interfaces for Virtual Environment and Teleoperator Systems March, 2005, Pisa, Italy, 195-201.

14. Gupta A. \& O’Malley M. K. (2006) Design of a haptic arm exoskeleton for training and rehabilitation. IEEE/ASME Transaction on Mechatronics 11: 280-289.

15. Kawasaki H., Ito S., Ishigure Y., Nishimoto Y., Aoki T., Mouri T., Sakaeda H. \& Abe M. (2007). Development of a hand motion assist robot for rehabilitation therapy by patient self-motion control. Proceedings of the IEEE International Conference on Rehabilitation Robotics June, 2007, Noordwijk, The Netherland, 234-240.

16. Rosen J., Brand N., Fuchs M. B. \& Arcan M. (2001). A myosignal-based powered exoskeleton system. IEEE Transaction on System, Man and Cybernetics, Part A
31(3): 210-222

17. Ball S.J., Brown I.E., \& Scott S.H. (2007). MEDARM: a rehabilitation robot with $5 \mathrm{DOF}$ at the shoulder complex. Proceedings of the IEEE/ASME International Conference on Advanced Intelligent Mechatronics September, 2007, Zurich, Swit zorland,1-6.

18. Carignan C., Liszka M. \& Roderick S. (2005). Design of an arm exoskeleton with scapula motion for shoulder rehabilitation. Proceedings of the International Conference on Advanced Robotics July, 2005, Seattle, USA, 524-531.

19. Farry K.A., Walker I.D. \& R.G. Baraniuk (1996). Myoelectric teleoperation of a complex robotic hand, IEEE Transactions on Robotics and Automation 12(5): 775-788.

20. Fukuda O., Tsuji T., Ohtsuka A. \& Kaneko M. (1998). EMG based human-robot interface for rehabilitation aid, Proceedings of the IEEE International Conference on Robotics and Automation, May, 1998, Leuren, Belgum, 3942-3947.

21. Nishikawa D., Yu W., Yokoi H. \& Kakazu Y. (1999). EMG prosthetic hand controller using real-time learning method. Proceedings of the IEEE International Conference on Systems, Man, and Cybernetics. October, 1999, Tokyo, Japan, 153-158.

22. O'Sullivan L.W. \& Gallwey T.J. (2002). Upper-limb surface electromyography at maximum supination and pronation torques: the effect of elbow and forearm angle. Journal of Electromyography and Kinesiology 12: 275-285.

23. Au A. T.C. \& Kirsch R.F. (2000). EMG-based prediction of shoulder and elbow kinematics in able-bodied and spinal cord injured individuals. IEEE Transactions on Rehabilitation Engineering 8(4): 471-480. 